\title{
A Note on the Evolution of the Whitney Sphere Along Mean Curvature Flow
}

\author{
Celso Viana ${ }^{1,2}$ (D)
}

Received: 24 April 2018 / Published online: 18 June 2020

(c) The Author(s) 2020

\begin{abstract}
We study the evolution of the Whitney sphere along the Lagrangian mean curvature flow. We show that equivariant Lagrangian spheres in $\mathbb{C}^{n}$ satisfying mild geometric assumptions collapse to a point in finite time and the tangent flows converge to a Lagrangian plane with multiplicity two.
\end{abstract}

Keywords Mean curvature flow · Lagrangian · Whitney sphere

Mathematics Subject Classification 53E10 - 53D12

\section{Introduction}

The Whitney sphere is the immersion $F: \mathbb{S}^{n} \rightarrow \mathbb{R}^{2 n}$ given by

$$
F\left(x_{1}, \ldots, x_{n+1}\right)=\frac{1}{1+x_{n+1}^{2}}\left(x_{1}, x_{1} x_{n+1}, \ldots, x_{n}, x_{n} x_{n+1}\right)
$$

This immersion is Lagrangian, i.e., $F^{*} \omega=0$, where $\omega$ is the standard symplectic form on $\mathbb{R}^{2 n}$. From the point of view of topology, the Whitney sphere is interesting since it has the best topological behavior: namely, it fails to be embedded only at the north and south pole where it has a transversal double point. An well known result of Gromov asserts that there are no embedded Lagrangian spheres in $\mathbb{C}^{n}$. On the geometry side, this immersion can be characterized by many geometric rigidity properties, see [3,12].

$\checkmark$ Celso Viana

celso@mat.ufmg.br

1 Union Building, University College London UCL, 25 Gordon Street, London WC1E 6BT, UK

2 Present Address: Departamento de Matemática, Universidade Federal de Minas Gerais (UFMG), Av Antônio Carlos 6627, Belo Horizonte 31270-901, Brazil 
In this sense, the Whitney sphere plays the role of totally umbilical hypersurfaces in $\mathbb{R}^{n}$ in the class of Lagrangian submanifolds.

Another interesting aspect of the Whitney sphere is that it appears as a limit surface under Lagrangian mean curvature flow of some well-behaved Lagrangian submanifolds in $\mathbb{R}^{4}$. Recall that the mean curvature flow (MCF) of an immersion $F_{0}: M^{k} \rightarrow \mathbb{R}^{m}$ is a map $F: M \rightarrow[0, T] \rightarrow \mathbb{R}^{m}$ such that $F(x, 0)=F_{0}$ and satisfies the equation

$$
\frac{\mathrm{d}}{\mathrm{d} t} F=H
$$

where $H$ is the mean curvature vector of $M^{n}$. It was shown by Smoczyk that the Lagrangian condition is preserved by MCF when the ambient space is a KählerEinstein manifold. The Lagrangian mean curvature flow gained a lot of interest recently as a potential tool to find minimal Lagrangian (special Lagrangian) in a given homology class or Hamiltonian isotopy class of a Calabi-Yau manifold. Special Lagrangian submanifolds have the remarkable property of being area minimizing by means of calibration arguments. The classical approach of minimizing area in a given class, however, does not seem very effective to find smooth special Lagrangian as shown by Schoen and Wolfson in [13].

Ideally, one could hope that the evolution of well behaved Lagrangian submanifolds along mean curvature flow to converge to special Lagrangians. In a series of works, A. Neves showed that finite time singularities are unavoidable in the Lagrangian mean curvature flow in general, see $[8,10]$. It is constructed in [8] a non-compact zero Maslov class Lagrangian in $\mathbb{R}^{4}$ with bounded Lagrangian angle and in the same Hamiltonian isotopy class of a Lagrangian plane that nevertheless develops a singularity in finite time. At the singular time the limit surface pictures like a connect sum of a smooth Lagrangian (diffeomorphic to a Lagrangian plane) with a Whitney sphere. Such construction were later generalized to 4-dimensional Calabi-Yau manifolds, see [10].

There are very few results regarding the evolution of compact Lagrangian submanifolds in $\mathbb{C}^{n}$. Motivate by this, we investigate the evolution of the Whitney sphere along mean curvature flow. Despite its many geometric properties, it is not a selfsimilar solution of the flow. By exploiting its rotationally symmetries, one can reduce its mean curvature flow to a flow about curves in the plane. As a particular case of our main result we prove

Let $F: \mathbb{S}^{n} \times[0, T) \rightarrow \mathbb{C}^{n}$ be the maximal existence mean curvature flow of the Whitney sphere. Then $F_{T}(x)=\{0\}$ for every $x \in \mathbb{S}^{n}$. The tangent flow at the origin is a Lagrangian plane with multiplicity two.

A Lagrangian submanifold $L \subset \mathbb{C}^{n}$ is called equivariant if there exists a antipodal invariant curve $\gamma: I \rightarrow \mathbb{C}$ such that $L$ can be written as

$$
L=\left\{\left(\gamma(u) G_{1}(x), \ldots, \gamma(u) G_{n}(x)\right) \in \mathbb{C}^{n}: G: \mathbb{C}^{n-1} \rightarrow \mathbb{R}^{n}\right\}
$$

where $G$ is a the standard embedding of $\mathbb{C}^{n-1}$ in $\mathbb{R}^{n}$. Using spherical coordinates on $\mathbb{C}^{n},(\cos (u) G(x), \sin (u))$, we check that the Whitney sphere is equivariant with 
Fig. 1 Whitney sphere

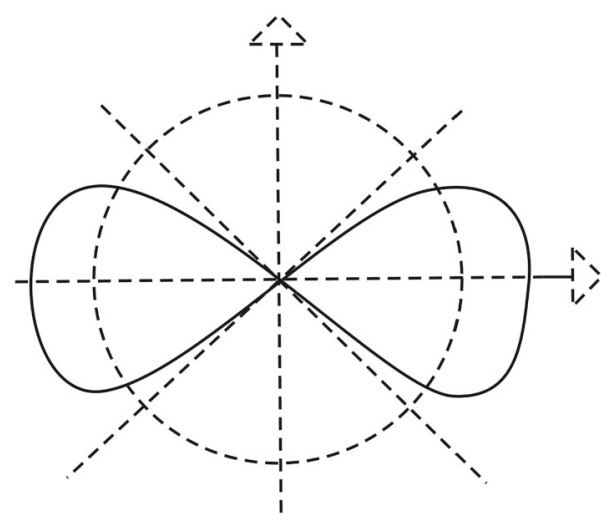

associated curve $\gamma_{0}:(0,2 \pi) \rightarrow \mathbb{R}^{2}$ given by:

$$
\gamma_{0}(u)=\left(\frac{\sin (u)}{1+\cos ^{2}(u)}, \frac{\sin (u) \cos (u)}{1+\cos ^{2}(u)}\right) .
$$

The equivariant property is preserved by the mean curvature flow and the corresponding evolution equation for $\gamma_{t}$ is

$$
\frac{\mathrm{d} \gamma}{\mathrm{d} t}=\vec{k}-(n-1) \frac{\gamma^{\perp}}{|\gamma|^{2}} .
$$

Here $\vec{k}$ denotes the curvature vector of $\gamma$, it is defined by $\vec{k}=\frac{1}{\left|\gamma^{\prime}\right|} \frac{\mathrm{d}}{\mathrm{d} u} \frac{\gamma^{\prime}}{\left|\gamma^{\prime}\right|}$, and $\gamma^{\perp}$ denotes the normal projection of the position vector $\gamma$. This flow is known as the equivariant flow.

Definition 1.1 Let $\mathcal{C}$ be the set of antipodal invariant figure eight curves $\gamma: \mathbb{C}^{1} \rightarrow \mathbb{C}$ with only one self-intersection which is transversal and located at the origin.

Definition 1.2 Let $\Omega_{\alpha}$ be the antipodal invariant region in $\mathbb{R}^{2}$ bounded by two lines through the origin with angle between them equal to $\alpha$ (Fig. 1).

Theorem 1.3 Let $\gamma$ be a curve in $\mathcal{C}$ satisfying at least one of the following assumptions:

(i) $\{\gamma\} \cap \mathbb{C}^{1}(R)$ has at most 4 points for every $R>0$;

(ii) $\{\gamma\} \subset \Omega \frac{\pi}{n}$.

If $\left\{\gamma_{t}\right\}_{t \in[0, T)}$ is the maximal equivariant flow of $\gamma$, then $\gamma_{T}=\{0\}$. Moreover, the tangent flow at the origin is a line with multiplicity two.

Remark 1.4 The assumptions in Theorem 1.3 are sharp. In Sect. 3 we construct for every $\alpha>\frac{\pi}{2}$ a curve $\gamma \in \mathcal{C}$ such that $\{\gamma\} \subset \Omega_{\alpha}$ that develops a non-trivial singularity at the origin along the equivariant flow (1.1) when $n=2$.

The proof of Theorem 1.3 follows closely the ideas in $[8,9]$ where it is shown that singularities for the mean curvature flow of monotone Lagrangian submanifolds in $\mathbb{R}^{4}$ are modeled on area minimizing cones. 


\section{Preliminaries}

Let $L$ be a Lagrangian submanifold in $\mathbb{C}^{n}$. This implies that $\left.\omega\right|_{L}=0$, where $\omega=$ $\sum_{i=1}^{n} \frac{\sqrt{-1}}{2} \mathrm{~d} z_{i} \wedge \overline{\mathrm{d} z_{i}}$ is the standard symplectic form on $\mathbb{C}^{n}$. Let $\Omega$ be the complex valued $n$-form given by

$$
\Omega=\mathrm{d} z_{1} \wedge \ldots \wedge \mathrm{d} z_{n}
$$

A standard computation implies that

$$
\left.\Omega\right|_{L}=e^{i \theta} \operatorname{vol}_{L}
$$

The multivalued function $\theta$ is called the Lagrangian angle of $L$. If $\theta$ is a single valued function, then $L$ is called zero-Maslov class. If $\theta=\theta_{0}$, then $L$ is calibrated by $\operatorname{Re}\left(e^{-i \theta_{0}} \Omega\right)$ and hence area-minimizing. In this case, $L$ is called special Lagrangian. More generally, the Lagrangian angle and the geometry of $L$ are related through $\vec{H}=J(\nabla \theta)$. Recall also the Liouville one form given by

$$
\lambda=\sum_{i=1}^{n} x_{i} \mathrm{~d} y_{i}-y_{i} \mathrm{~d} x_{i}
$$

One can check that $\mathrm{d} \lambda=\omega$. In particular, $[\lambda] \in H_{1}(L)$. When $[\lambda]=c[\mathrm{~d} \theta]$ for some $c \in \mathbb{R}$, then $L$ is said to be a monotone Lagrangian.

Let $L$ be a equivariant Lagrangian submanifold in $\mathbb{R}^{2 n}$. Hence, there exists a regular curve $\gamma$ in $\mathbb{R}^{2}$ such that

$$
L=\left\{\left(\gamma G_{1}, \ldots, \gamma G_{n}\right) \in \mathbb{R}^{2 n}, \sum_{i=1}^{n} G_{i}^{2}=1\right\}
$$

After choosing a parametrization of $\gamma$ we have

$$
\Omega_{L}:=\left.\mathrm{d} z_{1} \wedge \cdots \wedge \mathrm{d} z_{n}\right|_{L}=e^{i \theta} \operatorname{vol}_{L}=\frac{\gamma^{\prime}}{\left|\gamma^{\prime}\right|} \cdot\left(\frac{\gamma}{|\gamma|}\right)^{n-1} \operatorname{vol}_{L}
$$

where $z \cdot w$ denotes the standard multiplication of complex numbers; here we consider $\gamma$ as complex valued function. The Lagrangian angle relates to the geometry of L.

If $L_{t}$ is the mean curvature flow starting at $L$, then $L_{t}$ shares the same rotational symmetries of $L$, i.e., $\left.L_{t}=\left\{\gamma_{t} G_{1}, \ldots, \gamma_{t} G_{n}\right): G=\left(G_{1}, \ldots, G_{n}\right) \in \mathbb{C}^{n-1}\right\}$. Moreover,

$$
\frac{\mathrm{d} \gamma}{\mathrm{d} t}=\vec{k}-(n-1) \frac{\gamma^{\perp}}{|\gamma|^{2}} \text {. }
$$

Although the term $\frac{\gamma^{\perp}}{|\gamma|^{2}}$ is not well defined at the origin the quantity has its meaning even when a curve goes through the origin as we can see below. 
Lemma 2.1 Let $\gamma:[-a, a] \rightarrow \mathbb{R}^{2}$ a smooth regular curve such that $\gamma(0)=0$. Then

$$
\lim _{s \rightarrow 0} \frac{\gamma^{\perp}}{|\gamma|^{2}}(s)=\frac{1}{2} \vec{k}(0) .
$$

Proof Let us write the left hand side as

$$
\frac{\gamma^{\perp}}{|\gamma|^{2}}(s)=\frac{1}{|\gamma|^{2}}\left\langle\gamma, i \frac{\gamma^{\prime}}{\left|\gamma^{\prime}\right|}\right\rangle i \frac{\gamma^{\prime}}{\left|\gamma^{\prime}\right|}=\frac{s^{2}}{|\gamma|^{2}}\left\langle\frac{\gamma-s \gamma^{\prime}(0)}{s^{2}}, i \frac{\gamma^{\prime}}{\left|\gamma^{\prime}\right|}\right\rangle i \frac{\gamma^{\prime}}{\left|\gamma^{\prime}\right|}
$$

Using that $\lim _{s \rightarrow 0} \frac{\gamma(s)}{s}=\gamma^{\prime}(0)$ and applying the L'Hopital's rule twice, we obtain

$$
\lim _{s \rightarrow 0} \frac{\gamma^{\perp}}{|\gamma|^{2}}(s)=\frac{1}{2} \frac{1}{\left|\gamma^{\prime}(0)\right|^{2}}\left\langle\gamma^{\prime \prime}(0), i \frac{\gamma^{\prime}(0)}{\left|\gamma^{\prime}\right|(0)}\right\rangle i \frac{\gamma^{\prime}(0)}{\left|\gamma^{\prime}(0)\right|}=\frac{1}{2} \vec{k}(0) .
$$

Proposition 2.2 (Neves [10]) Let $\gamma_{i, t}:[-a, a] \rightarrow \mathbb{R}^{2}, i=1,2$ and $0 \leq t \leq T$, smooth regular curves satisfying

(1) $\gamma_{i, t}(-s)=-\gamma_{i, t}(s)$ for all $0 \leq t \leq T$ and for every $s \in[-a, a]$.

(2) The curve $\gamma_{i, t}, i=1,2$, solves the equation

$$
\frac{\mathrm{d} \gamma}{\mathrm{d} t}=\vec{k}-(n-1) \frac{\gamma^{\perp}}{|\gamma|^{2}}
$$

(3) $\gamma_{1,0} \cap \gamma_{2,0}=\{0\}$ (non-tangential intersection) and $\partial \gamma_{1, t} \cap \gamma_{2, t}=\partial \gamma_{2, t} \cap \gamma_{1, t}=\varnothing$ for all $t$.

Then for all $0 \leq t \leq T$ we have $\gamma_{1, t} \cap \gamma_{2, t}=\{0\}$.

Proof It suffices to restrict to what happens near the origin since the proposition follows from the standard maximum principle applied to the first time of tangential intersection.

First notice that $\gamma_{i, t}$ can be written as a graph on $[-\delta, \delta]$ for some $\delta>0$. Hence, $\gamma_{i, t}(s)=\left(s, f_{i, t}(s)\right)$ and we define $h_{i, t}(s)=\frac{f_{i, t}(s)}{s}$. Let's check that $h_{i, t}(s)$ is smooth: if $s \neq 0$, then

$$
\begin{aligned}
h^{\prime}(s)=\frac{f^{\prime} s-f}{s^{2}} \text { and } h^{\prime \prime}(s) & =\frac{\left(f^{\prime \prime} s+f^{\prime}-f^{\prime}\right) s^{2}-\left(f^{\prime} s-f\right) 2 s}{s^{4}} \\
& =\frac{f^{\prime \prime}}{s}+2 \frac{f-f^{\prime} s}{s^{3}}
\end{aligned}
$$

Since $f(0)=0$ and $f^{\prime \prime}(0)=0$ (item (1)), we can apply L'Hopital's rule to show that $\alpha^{\prime}$ and $\alpha^{\prime \prime}$ in (2.5) have a limit when $s \rightarrow 0$. Hence, $h$ is twice differentiable. 
Finally we consider the function $u_{t}(s)=h_{1, t}-h_{2, t}$. Notice that $u_{0}>0$ by assumption (3) and $u_{t}(s)=u_{t}(-s)$. Recall that in the case of a graph $\gamma(s)=(s, f(s))$ we have

$$
\gamma^{\prime}=\left(1, f^{\prime}\right), \quad v=\frac{\left(f^{\prime},-1\right)}{\sqrt{1+\left(f^{\prime}\right)^{2}}} \text { and } \vec{k}=-\frac{f^{\prime \prime}}{\left(1+\left(f^{\prime}\right)^{2}\right)^{\frac{3}{2}}} v .
$$

Besides,

$$
\frac{\gamma^{\perp}}{|\gamma|^{2}}=\frac{s f^{\prime}-f}{s^{2}+f^{2}} \frac{1}{\sqrt{1+\left(f^{\prime}\right)^{2}}} \nu
$$

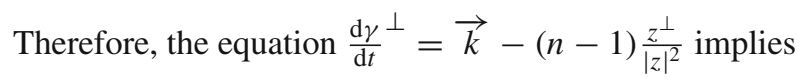

$$
\frac{\mathrm{d} f}{\mathrm{~d} t}=\frac{f^{\prime \prime}}{1+\left(f^{\prime}\right)^{2}}+(n-1)\left(\arctan \frac{f}{s}\right)^{\prime} .
$$

Standard computations imply that $h_{i, t}=\frac{f_{i, t}}{s}$ satisfies

$$
\frac{\mathrm{d} h_{i, t}}{\mathrm{~d} t}=\frac{h_{i, t}^{\prime \prime}}{1+\left(s h_{i, t}^{\prime}+h_{i, t}\right)^{2}}+\frac{h_{i, t}^{\prime}}{s} \frac{2}{1+\left(s h_{i, t}^{\prime}+h_{i, t}\right)^{2}}+(n-1) \frac{h_{i, t}^{\prime}}{s} \frac{1}{1+h_{i, t}^{2}} .
$$

Now we proceed to find the equation for $\frac{\mathrm{d} u_{t}}{\mathrm{~d} t}$. Using that $\frac{h_{i, t}}{\mathrm{~s}}$ is also smooth, one can checked that

$$
\frac{\mathrm{d} u_{t}}{\mathrm{~d} t}=C_{1}^{2} u_{t}^{\prime \prime}+C_{2} u_{t}^{\prime}+C_{3} u_{t}+C_{4}^{2} \frac{u_{t}^{\prime}}{s},
$$

where each $C_{k}$ is a smooth and bounded function. By item (3), the function $u_{t=0}$ is strictly positive since $\gamma_{1}$ and $\gamma_{2}$ have a non-tangential intersection at the origin.

Suppose $T_{1}$ is the first time where $u_{t}$ has a zero say at $s_{0}$. Hence, $s_{0}$ is a minimum point as $u_{T_{1}} \geq 0$. We consider the function $v_{t}=u_{t} e^{-C t}+\varepsilon\left(t-T_{1}\right)$ where $C$ is very large and $\varepsilon$ is a very small positive number. So at $\left(s_{0}, T_{1}\right)$ we have

$$
0 \geq \frac{\mathrm{d} v_{t}}{\mathrm{~d} t}\left(s_{0}, T_{1}\right)=\frac{\mathrm{d} u_{t}}{\mathrm{~d} t}\left(s_{0}, T_{1}\right) e^{-C T_{1}}+\varepsilon \geq \varepsilon+C_{4}^{2} \frac{u_{t}^{\prime}\left(s_{0}\right)}{s_{0}} e^{-C T_{1}}
$$

We used in the equality part that $u_{T_{1}}(s)=0$ and that $u_{T_{1}}^{\prime}\left(s_{0}\right)=0$ and $u_{T_{1}}^{\prime \prime}(s) \leq 0$ since $s_{0}$ is a minimum point for $u_{T_{1}}$. If $s_{0} \neq 0$ then the second term in the right hand side is zero and we get a contradiction. If $s_{0}=0$ then that term is just $u_{t}^{\prime \prime}(0) e^{-C T_{1}}$ by the L'Hopital's rule, hence, non-negative and we obtain a contradiction again.

Corollary 2.3 The set $\mathcal{C}$ is preserved by the equivariant flow. Moreover, if $\gamma \in \mathcal{C}$ satisfies item (i) (respectively, item (ii)) in Theorem 1.3, then so does $\gamma_{t}$. 
Proof The symmetries of the curve $\gamma$ are preserved by the equivariant flow, hence $\gamma_{t}$ is also antipodal invariant. Proposition 2.2 guarantees that the only self intersection of $\gamma_{t}$ is at the origin and it is transversal for every $t$. Hence, $\mathcal{C}$ is preserved by the equivariant flow. Moreover, Proposition 2.2 also implies that $\gamma_{t}(s)$ can only intersect the line $s e^{\overrightarrow{i \beta}}$ s $\mathbb{R}$, with $\frac{\pi}{n}<\beta<\pi-\frac{\pi}{n}$, only at the origin for every $t \in[0, T)$. Therefore, if $\{\gamma\} \subset \Omega_{\frac{\pi}{n}}$, then $\left\{\gamma_{t}\right\} \subset \Omega_{\frac{\pi}{n}}$ also. Finally, by Theorem 1.3 in [2], the number of intersections between $\{\gamma\}$ and $\mathbb{C}^{1}(R)$ is non-increasing along the flow.

Lemma 2.4 If $\gamma \in \Omega_{\frac{\pi}{n}}$, then for every $t>0$ there exists $\delta_{t}>0$ such that $\left\{\gamma_{t}\right\} \subset$ $\Omega \frac{\pi}{n}-\delta_{t}$.

Proof Since $\gamma \in \mathcal{C}$ is antipodal invariant and passes through the origin, one can check that $\lim _{s \rightarrow 0} \frac{\gamma^{\perp}}{|\gamma|^{2}}(s)=0$, where $\gamma(s)$ is a local parametrization of $\gamma$ with $\gamma(s)=$ $-\gamma(-s)$. By Lemma 2.1, we have that $\vec{k}\left(z_{0}\right)=\vec{k}\left(-z_{0}\right)=0$, where $\gamma\left(z_{0}\right)=$ $\gamma\left(-z_{0}\right)=0$. Consequently, $\vec{H}\left(z_{0}\right)=\vec{H}\left(-z_{0}\right)=0$. This implies that $z_{0}$ and $-z_{0}$ are critical points of the Lagrangian angle $\theta_{L}$. It can be check easily that they correspond to local minimum and local maximum critical points. The strong maximum principle applied to $\frac{\mathrm{d}}{\mathrm{d} t} \theta=\Delta \theta$ implies that $\theta_{t}\left(z_{0}\right)<\theta\left(z_{0}\right)$ and $\theta_{t}\left(-z_{0}\right)>\theta\left(-z_{0}\right)$.

Let us use $\operatorname{Area}(\gamma)$ to denote the area enclosed by $\gamma \in \mathcal{C}$. By the Stokes' theorem we have that $\operatorname{Area}\left(\gamma_{t}\right)=-\frac{1}{2} \int_{\gamma_{t}}\left\langle\gamma_{t}, v\right\rangle \mathrm{d}_{\gamma_{t}}$, where $v$ is the unit outward normal vector of $\gamma$.

\section{Lemma 2.5}

$$
\pi(T-t) \leq \operatorname{Area}\left(\gamma_{t}\right)-\operatorname{Area}\left(\gamma_{T}\right) \leq 3 \pi(T-t) .
$$

Proof Let $\gamma_{t}(u)$ be a parametrization of $\gamma_{t}$. Using that $v=i \frac{\gamma_{t}^{\prime}}{\left|\gamma_{t}^{\prime}\right|}$, we have that $\operatorname{Area}\left(\gamma_{t}\right)=-\frac{1}{2} \int_{\gamma_{t}}\left\langle\gamma_{t}, i \gamma_{t}^{\prime}\right\rangle \mathrm{d} u$. Hence,

$$
\begin{aligned}
\operatorname{Area}^{\prime}(t) & =-\frac{1}{2} \int_{\gamma_{t}}\left(\left\langle\partial_{t} \gamma, i \gamma_{t}^{\prime}\right\rangle+\left\langle\gamma, i\left(\partial_{t} \gamma\right)^{\prime}\right\rangle\right) \mathrm{d} u \\
& =-\frac{1}{2} \int_{\gamma_{t}}\left(\left\langle\partial_{t} \gamma, i \gamma_{t}^{\prime}\right\rangle+\left\langle\gamma_{t}, i \partial_{t} \gamma\right\rangle^{\prime}-\left\langle\gamma^{\prime}, i \partial_{t} \gamma\right\rangle\right) \mathrm{d} u \\
& =-\int_{\gamma_{t}}\left\langle\partial_{t} \gamma, i \gamma_{t}^{\prime}\right\rangle \mathrm{d} u-\frac{1}{2} \int_{\gamma_{t}}\left\langle\gamma_{t}, i \partial_{t} \gamma\right\rangle^{\prime} \mathrm{d} u=-\int_{\gamma_{t}}\left\langle\partial_{t} \gamma, \nu\right\rangle \mathrm{d} \gamma_{t} .
\end{aligned}
$$

The last equality follows from the Fundamental Theorem of Calculus. Hence,

$$
\operatorname{Area}^{\prime}(t)=-\int_{\gamma_{t}}\left\langle\vec{k}-(n-1) \frac{z^{\perp}}{|z|^{2}}, v\right\rangle \mathrm{d}_{\gamma_{t}}=-\int_{\gamma_{t}}\langle\vec{k}, v\rangle \mathrm{d}_{\gamma_{t}} .
$$

The last equality follows from the Divergence Theorem applied to vector field $X=\frac{z}{|z|^{2}}$ and the fact that $z=0$ is not in the interior of the region enclosed by $\gamma_{t}$. Combining 
the Gauss-Bonnet theorem and the fact that the exterior angle $\alpha_{t}$ of $\gamma_{t}$ at the origin is in $[-\pi, \pi]$ we obtain

$$
\int_{\gamma_{t}}\langle\vec{k},-v\rangle \mathrm{d}_{\gamma_{t}}+\alpha_{t}=2 \pi \Longrightarrow \pi \leq \int_{\gamma_{t}}\langle\vec{k},-v\rangle \mathrm{d}_{\gamma_{t}} \leq 3 \pi .
$$

Therefore, $-3 \pi \leq \operatorname{Area}^{\prime}\left(\gamma_{t}\right) \leq-\pi$. The Lemma now follows if we integrate this quantity from $t$ to $T$.

\section{Proof of the Theorem}

Let $L_{t}$ be a solution of the mean curvature flow starting on a $k$-dimensional submanifold $L$ in $\mathbb{R}^{m}$. Consider the backward heat kernel

$$
\Phi_{x_{0}, T}(x, t)=\frac{1}{(4 \pi(T-t))^{\frac{k}{2}}} e^{-\frac{\left|x-x_{0}\right|^{2}}{4(T-t)}} .
$$

The following formula is known as the Huisken's monotonicity formula:

$$
\begin{aligned}
& \frac{\mathrm{d}}{\mathrm{d} t} \int_{L_{t}} f_{t} \Phi_{x_{0}, T} \mathrm{~d} \mathcal{H}^{k} \\
& \quad=\int_{L_{t}}\left(\frac{\mathrm{d}}{\mathrm{d} t} f_{t}-\Delta f_{t}-\left|H-\frac{\left(x-x_{0}\right)^{\perp}}{2(T-t)}\right|^{2} f_{t}\right) \Phi_{x_{0}, T} \mathrm{~d} \mathcal{H}^{k}
\end{aligned}
$$

where $d \mathcal{H}^{k}$ denotes the $k$-dimensional Hausdorff measure.

Recall that if $\left\{L_{t}\right\}_{t \in[0, T)}$ is the Lagrangian mean curvature flow starting at $L$, then

$$
L_{s}^{\sigma}=\sigma\left(L_{T+\frac{s}{\sigma^{2}}}-x_{0}\right)
$$

for $s \in\left[-T \lambda^{2}, 0\right)$, also satisfies the Lagrangian mean curvature flow and is referred as the tangent flow at $x_{0}$. The following is a restatement of Theorem 1.3:

Theorem 3.1 Let $\gamma$ be a curve in $\mathcal{C}$ which satisfies at least one of the following assumptions

(i) $\{\gamma\} \cap \mathbb{C}^{1}(R)$ has at most 4 points for every $R>0$;

(ii) $\{\gamma\} \subset \Omega \frac{\pi}{n}$.

If $\left\{\gamma_{t}\right\}_{t \in[0, T)}$ is the maximal equivariant flow of $\gamma$, then $\gamma_{T}=\{0\}$. Moreover, the tangent flow at the origin is a line with multiplicity two.

Proof Let us prove first that if $z=0$ is a singular point, then $\gamma_{T}=\{0\}$. Arguing by contradiction, we assume that $z=0$ is a singular point for $\left\{\gamma_{t}\right\}_{0 \leq t<T}$ and $\gamma_{T} \neq\{0\}$. Given $\sigma_{i} \rightarrow \infty$, let $\gamma_{s}^{i}=\sigma_{i} \gamma_{T+\frac{s}{\sigma_{i}^{2}}}$. 
Lemma 3.2 Let $a$ and $b$ real numbers such that $a<b<0$. Then

$$
\lim _{i \rightarrow \infty} \int_{a}^{b} \int_{\gamma_{s}^{i} \cap A\left(\frac{1}{\eta}, \eta, 0\right)}\left(|\vec{k}|^{2}+\left|\gamma^{\perp}\right|^{2}\right) \mathrm{d} \mathcal{H}^{1} d s=0
$$

where $A\left(\frac{1}{\eta}, \eta, 0\right)$ is an annulus centered at $z=0$ with inner and outer radius $\eta$ and $\frac{1}{\eta}$, respectively.

Proof Let $L_{s}^{i}$ be the immersed Lagrangian sphere in $\mathbb{C}^{2}$ obtained via $L_{s}^{i}=$ $\left(\gamma_{s}^{i} G_{1}, \ldots, \gamma_{s}^{i} G_{n}\right)$. It is proved in Lemma 5.4 in [8] that

$$
\lim _{i \rightarrow \infty} \int_{a}^{b} \int_{L_{s}^{i} \cap B_{R}(0)}\left(|H|^{2}+\left|x^{\perp}\right|^{2}\right) \mathrm{d} \mathcal{H}^{n}(x) d s=0
$$

where $H$ is the mean curvature vector of $L_{s}^{i}$. For the convenience of the reader let us recall the proof of this fact. It is a standard computation to check that the Lagrangian angle $\theta$ obeys the following evolution equation $\frac{\mathrm{d}}{\mathrm{d} t} \theta_{i, s}^{2}=\Delta \theta_{i, s}^{2}-2|H|^{2}$. Applying (3.1) with $f_{t}=\theta_{i, s}^{2}$ and $f_{t}=1$, we obtain

$$
\begin{aligned}
\frac{\mathrm{d}}{\mathrm{d} s} \int_{L_{s}^{i}} \theta_{i, s}^{2} \Phi \mathrm{d} \mathcal{H}^{n} & =\int_{L_{s}^{i}}\left(-2|H|^{2}-\left|H-\frac{x^{\perp}}{2 s}\right|^{2} \theta_{i, s}^{2}\right) \Phi \mathrm{d} \mathcal{H}^{n} \\
\frac{\mathrm{d}}{\mathrm{d} s} \int_{L_{s}^{i}} \Phi \mathrm{d} \mathcal{H}^{n} & =\int_{L_{s}^{i}}-\left|H-\frac{x^{\perp}}{2 s}\right|^{2} \Phi \mathrm{d} \mathcal{H}^{n},
\end{aligned}
$$

respectively. Integrating (3.3) from $a$ to $b$ gives

$$
2 \lim _{i \rightarrow \infty} \int_{a}^{b} \int_{L_{s}^{i}}|H|^{2} \Phi \mathrm{d} \mathcal{H}^{n} d s \leq \lim _{i \rightarrow \infty} \int_{L_{b}^{i}} \theta_{i, b}^{2} \Phi \mathrm{d} \mathcal{H}^{n}-\lim _{i \rightarrow \infty} \int_{L_{a}^{i}} \theta_{i, a}^{2} \Phi \mathrm{d} \mathcal{H}^{n}=0 .
$$

The last inequality follows from the scale invariance and monotonicity of $\int_{L_{t}} \theta^{2} \Phi \mathrm{d} \mathcal{H}^{n}$. Similarly, we obtain

$$
\lim _{i \rightarrow \infty} \int_{a}^{b} \int_{L_{s}^{i}}\left|H-\frac{x^{\perp}}{2 s}\right|^{2} \Phi \mathrm{d} \mathcal{H}^{n} d s=\lim _{i \rightarrow \infty} \int_{L_{b}^{i}} \Phi \mathrm{d} \mathcal{H}^{2}-\lim _{i \rightarrow \infty} \int_{L_{a}^{i}} \Phi \mathrm{d} \mathcal{H}^{n}=0
$$

It follows from the triangular inequality that

$$
\lim _{i \rightarrow \infty} \int_{a}^{b} \int_{L_{s}^{i}}\left|\frac{x^{\perp}}{2 s}\right|^{2} \Phi \mathrm{d} \mathcal{H}^{n} \mathrm{~d} s=0
$$


This completes the proof of (3.2). As $|H|^{2}=\left|\vec{k}-(n-1) \frac{\gamma^{\perp}}{|\gamma|^{2}}\right|^{2}$ and $\left|x^{\perp}\right|^{2}=\left|\gamma^{\perp}\right|^{2}$, we obtain for each $\eta>0$ that

$$
\lim _{i \rightarrow \infty} \int_{a}^{b} \int_{\gamma_{s}^{i} \cap A\left(\frac{1}{\eta}, \eta, 0\right)}\left(|\vec{k}|^{2}+\left|\gamma^{\perp}\right|^{2}\right) \mathrm{d} \mathcal{H}^{1} \mathrm{~d} s=0
$$

$\square$ From previous lemma it follows that for almost every $s \in(a, b)$ that

$$
\lim _{i \rightarrow \infty} \int_{\gamma_{s}^{i} \cap A\left(\frac{1}{\eta}, \eta, 0\right)}\left(|\vec{k}|^{2}+\left|\gamma^{\perp}\right|^{2}\right) \mathrm{d} \mathcal{H}^{1}=0 .
$$

This implies that $\gamma_{s}^{i}$ converges to a union of lines in $C_{l o c}^{1, \frac{1}{2}}\left(\mathbb{R}^{2}-\{0\}\right)$. In fact, each connected component of $\gamma_{s}^{i}$ inside $B_{R}(0)-\{0\}$ converge to a line segment with multiplicity one since the convergence is in $C_{l o c}^{1, \frac{1}{2}}\left(\mathbb{R}^{2}-\{0\}\right)$.

Assume first that $\gamma$ satisfies item i), then by Proposition 2.2 and Corollary 2.3, the curve $\gamma_{s}^{i}$ in $B_{R}(0)-\{0\}$ has two embedded connected components. Hence, each converges to a line segment with multiplicity one in $B_{R}(0)-\{0\}$. Equivalently, in a neighborhood of the origin $L_{t}$ is a union of two smooth embedded discs intersecting transversally at a interior point. Hence, each piece of $L_{s}^{i}$ converges weakly to a plane with multiplicity one. Since $\gamma_{T} \neq\{0\}$, we can talk about the localized Gaussian density of each connected component of $L_{t} \cap B_{r}(0)$ computed at $(0, T)$ which will be very close to one. Applying White's Local Regularity Theorem, see localized version Theorem 5.6 in [4]), to each component of $L_{t} \cap B_{r}(0)$, we conclude that the origin is not a singularity of $\left\{L_{t}\right\}_{t \in[0, T)}$, contradiction.

To handle other connected components of $\gamma_{s}^{i}$ in $B_{4 R}(0)$ we study the Lagrangian angle $\theta_{s}^{i}$. Let $\beta$ be a primitive of $\lambda_{L}$. It is proved in [9] that $\nabla \beta=J\left(x^{\perp}\right)$ and $\frac{\mathrm{d}}{\mathrm{d} t} \beta=$ $\Delta \beta-2 \theta$. This implies that the function $u=\beta+2\left(t-t_{0}\right) \theta$ satisfies $\frac{\mathrm{d}}{\mathrm{d} t} f(u)=$ $\Delta f(u)-f^{\prime \prime}(u)\left|x^{\perp}+2\left(t-t_{0}\right) H\right|^{2}$, where $f \in C_{0}^{\infty}(\mathbb{R})$. Plugging the function $f(u)$ in (3.1), we obtain

$$
\frac{\mathrm{d}}{\mathrm{d} s} \int_{L_{s}^{i}} f\left(u_{s}^{i}\right) \Phi=-\int_{L_{s}^{i}}\left|H-\frac{x^{\perp}}{2 s}\right|^{2} f\left(u_{s}^{i}\right) \Phi+f^{\prime \prime}\left(u_{s}^{i}\right)\left|x^{\perp}+2\left(s-s_{0}\right) H\right|^{2} \Phi .
$$

Integrating this formula from -1 to $s_{0}$ and using (3.2), we obtain

$$
\lim _{i \rightarrow \infty} \int_{L_{s_{0}}^{i} \cap B_{4 R}(0)} f\left(\beta_{s_{0}}^{i}\right) \Phi=\lim _{i \rightarrow \infty} \int_{L_{-1}^{i} \cap B_{4 R}(0)} f\left(\beta_{-1}^{i}-2\left(1+s_{0}\right) \theta_{-1}^{i}\right) \Phi .
$$

Let $\gamma^{i}$ be a connected component of $\gamma_{s}^{i}$ in $B_{4 R}(0)$ that intersects $B_{R}(0)$ and does not passes through the origin. Since $\left|\nabla f\left(\beta_{s}^{i}\right)\right|$ is bounded, there exists a constant $b_{s_{0}}$ such that $\lim _{i \rightarrow \infty} f\left(\beta_{s_{0}}^{i}\right)=f\left(b_{s_{0}}\right)$. Similarly, $\lim _{i \rightarrow \infty} f\left(\beta_{-1}^{i}\right)=f\left(b_{-1}\right)$. As before, $\gamma_{i}$ converges in $C^{1, \frac{1}{2}}\left(\mathbb{R}^{2}-\{0\}\right)$ to lines $l_{v_{1}^{s}}$ and $l_{v_{2}^{s}}$ in the direction of the vectors $\overrightarrow{v_{i}^{s}}$. Moreover, 


$$
\lim _{i \rightarrow \infty} \int_{\gamma^{i}} f\left(\beta_{-1}^{i}-2\left(1+s_{0}\right) \theta_{-1}^{i}\right) \Phi \mathrm{d} \mathcal{H}^{1}=\sum_{i=1}^{2} \int_{l_{\vec{v}_{i}}} f\left(b_{-1}-2\left(1+s_{0}\right) \theta_{i}\right) \Phi \mathrm{d} \mathcal{H}^{1}
$$

Note that (2.3) implies that $\theta_{s}^{i}$ converge to a constant in each connected component of $\gamma_{s}^{i} \cap\left(B_{R}(0)-B_{r}(0)\right)$. We claim that $\theta_{1}=\theta_{2}$. Otherwise, by choosing $f$ with support near $b_{s_{0}}$ and equal to 1 near $b_{s_{0}}$, we obtain

$$
\sum_{i=1}^{2} \int_{l_{\overrightarrow{v_{i}}} \cap B_{R}(0)} \Phi \mathrm{d} \mathcal{H}^{1}=\int_{l_{\overrightarrow{v_{0}}} \cap B_{R}(0)} \Phi \mathrm{d} \mathcal{H}^{1}
$$

contradiction.

Let us assume that $\gamma$ satisfies item (ii). In this case, $\gamma_{s}^{i} \cap B_{4 R}(0)$ has a connected component $\gamma^{i}$ intersecting $B_{2 R}$ which converges in $C^{1, \frac{1}{2}}\left(B_{R}(0)-\{0\}\right)$ to the lines $\gamma_{A}$ and $\gamma_{B}$ with multiplicity one. Moreover, $\theta_{S}^{i}$ converge to a constant $\theta_{0}$ on each connected component of $\gamma^{i} \cap\left(B_{R}(0)-B_{r}(0)\right)$. This implies that $\gamma_{A}=\gamma_{B}$ with the same orientation or the angle between $\gamma_{A}$ and $\gamma_{B}$ is $\frac{\pi}{n}$. The first case cannot happen since $I_{2}\left(\beta_{s}^{i}, \mathbb{C}^{1}(0, r)\right)=0$, where $I_{2}(\cdot, \cdot)$ is the intersection number mod 2 . The second case cannot happen since $\left\{\gamma_{t}\right\} \subset \Omega_{\frac{\pi}{n}-\delta_{t}}$ by Lemma 2.4. Hence, the origin is not a singularity if we assume that $\gamma_{T} \neq\{0\}^{n}$.

On the other hand, no singularities away from the origin occur. Indeed, in [11] Oaks complement the work of Angenent on singularities of equations of type $\frac{\mathrm{d}}{\mathrm{d} t} \gamma_{t}=$ $V(\vec{T}, k) \vec{N}$ by showing that near the singularity the curve $\gamma_{t}$ must lose a self intersection. Since Proposition 2.2 asserts the only self intersection of $\gamma_{t}$ is at the origin we are done.

Now let us prove that the tangent flow at the singular point is a line through the origin with multiplicity 2 . For this we choose a sequence of scale factors $\lambda_{i} \rightarrow+\infty$ and we set $\gamma_{s}^{i}=\lambda_{i} \gamma_{T+\frac{s}{\lambda_{i}^{2}}}$ defined in $\left[-T \lambda_{i}^{2}, 0\right)$.

As discussed before $\gamma_{s}^{i}$ converges in $C_{\text {loc }}^{1, \frac{1}{2}}\left(\mathbb{R}^{2}-\{0\}\right)$ to a union of two lines through the origin for almost every $s$ fixed. Let us denote them by $l_{A}$ and $l_{B}$. As $\operatorname{Area}\left(\gamma_{t}\right)$ is going to zero there exist a unique $t_{i} \in[0, T)$ for which Area $\left(\gamma_{t_{i}}\right)=\frac{1}{\lambda_{i}^{2}}$. This implies that $\operatorname{Area}\left(\gamma_{s_{1}^{i}}^{i}\right)=1$, where $s_{1}^{i}$ is given by $s_{1}^{i}=-\lambda_{i}^{2}\left(T-t_{i}\right)$. Since $\pi(T-t) \leq A(t) \leq$ $3 \pi(T-t)$ by Lemma 2.5, we obtain that $s_{1}^{i} \in\left[-\frac{1}{\pi},-\frac{1}{3 \pi}\right]$. In particular, if $s^{*}=-\frac{1}{3 \pi}$, then $\limsup _{i \rightarrow \infty} \operatorname{Area}\left(\gamma_{S^{*}}^{i}\right) \leq 1$. Therefore, $\gamma_{S^{*}}^{i}$ must converge to $2 \gamma_{A}+2 \gamma_{B}$ or $\gamma_{A}=\gamma_{B}$ since $\gamma_{s^{*}}^{i}$ is becoming non-compact enclosing bounded area.

The first case does not happen as it violates the assumptions (i) and (ii) as discussed above.

The next example constructs equivariant Lagrangian spheres in $\mathbb{R}^{4}$ that do not collapse to a point along the mean curvature flow.

Example 3.3 Let $\gamma_{0}$ be the curve $\gamma^{\alpha}(u)=\sin \left(\frac{\pi u}{\alpha}\right)^{-\frac{\alpha}{\pi}}(\cos (u), \sin (u))$ with $u \in \mathbb{R}$. The existence of a solution of the equivariant flow starting at $\gamma^{\alpha}$ is given in [8], let 
Fig. 2 Curve $\beta$

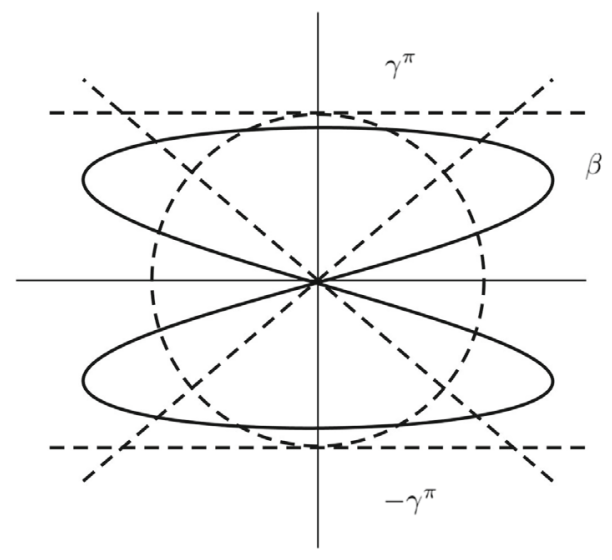

us denote it by $\left\{\gamma_{t}\right\}_{t \in\left[0, T_{\alpha}\right)}$. It is shown in [8] that when $\alpha>\frac{\pi}{2}$, then $T_{\alpha}<\infty$ and $\gamma_{t}$ develops a singularity at the origin. When $\alpha \in(0, \pi)$, then $\gamma^{\alpha}$ is contained in $\Omega_{\alpha}$ and it is asymptotic to its boundary. Consider the region $U_{\alpha}$ in $\Omega_{\alpha}$ that is bounded by $\left\{\gamma^{\alpha}\right\} \cup\left\{-\gamma^{\alpha}\right\}$. One can check that $U_{\alpha}$ has infinite area. Choose $\beta \in \mathcal{C}$ contained in $U_{\alpha}$ whose area enclosed, $\operatorname{Area}(\beta)$, is greater than $3 \pi T_{\alpha}$. See Fig. 2 for the case $\alpha=\pi$. Let $\left\{\beta_{t}\right\}_{t \in[0, T)}$ be the solution of the equivariant flow starting at $\beta$. By the avoidance principle, $\beta_{t}$ and $\gamma_{t}$ do not intersect. Hence, $T<T_{\alpha}$. On the other hand, by Lemma 2.5 we have that $\operatorname{Area}\left(\beta_{T}\right) \geq \operatorname{Area}(\beta)-3 \pi T \geq 3 \pi\left(T_{\alpha}-T\right)>0$. Therefore, a non trivial singularity must occur at the origin.

Let us show that any Type II dilation of $\gamma_{t}$ near the singularity converges to an eternal solution of curve shortening flow. As in Chapter 4 in [7], there exist for each $k>0$, points $z_{k} \in \gamma_{t}\left(\mathbb{C}^{1}\right), t_{k} \in\left[0, T-\frac{1}{k}\right]$, and scaling $\lambda_{k}>0$ such that $\beta_{s}^{k}=\lambda_{k}\left(\gamma_{T+\frac{s}{\lambda_{k}^{2}}}-z_{k}\right)$ satisfies

$$
\frac{\mathrm{d}}{\mathrm{d} s} \beta_{s}^{k}=\vec{k}\left(\beta_{s}^{k}\right)-(n-1) \frac{\left(\beta_{s}^{k}+\lambda_{k} z_{k}\right)^{\perp}}{\left|\beta_{s}^{k}+\lambda_{k} z_{k}\right|^{2}},
$$

where $s \in\left(a_{k}, b_{k}\right)$. Moreover, $\lim _{k \rightarrow \infty} a_{k}=-\infty, \lim _{k \rightarrow \infty} b_{k}=\infty$, and $0<$ $\lim _{k \rightarrow \infty} \sup _{\left(a_{k}, b_{k}\right) \times \mathbb{C}^{1}}\left|\vec{k}\left(\beta_{s}^{k}\right)\right| \leq C$. It is proved that $\beta_{s}^{k}$ converge smoothly as $k \rightarrow$ $\infty$ to a non-compact flow $\left(\beta_{s}\right)_{s \in \mathbb{R}}$. We claim that $\lim _{k \rightarrow \infty} \lambda_{k} z_{k}=\infty$. If not, then we could replace the points $z_{k}$ by $z=0$ and obtain the same conclusion. This is impossible since central dilations converge to lines. Therefore, as $k \rightarrow \infty$,

$$
\frac{\mathrm{d}}{\mathrm{d} s} \beta_{s}=\vec{k}\left(\beta_{s}\right)
$$

Acknowledgements I would like to thank Jason Lotay for suggesting this problem and for his encouragement and support during this work. I also thank my advisor André Neves for many helpful conversations. This work was supported by the Engineering and Physical Sciences Research Council [EP/L015234/1], and the EPSRC Centre for Doctoral Training in Geometry and Number Theory (London School of Geometry and Number Theory), University College London. 
Open Access This article is licensed under a Creative Commons Attribution 4.0 International License, which permits use, sharing, adaptation, distribution and reproduction in any medium or format, as long as you give appropriate credit to the original author(s) and the source, provide a link to the Creative Commons licence, and indicate if changes were made. The images or other third party material in this article are included in the article's Creative Commons licence, unless indicated otherwise in a credit line to the material. If material is not included in the article's Creative Commons licence and your intended use is not permitted by statutory regulation or exceeds the permitted use, you will need to obtain permission directly from the copyright holder. To view a copy of this licence, visit http://creativecommons.org/licenses/by/4.0/.

\section{References}

1. Angenent, S.: Parabolic equations for curves on surfaces. I. Curves with p-integrable curvature. Ann. Math. (2) 132, 451-483 (1990)

2. Angenent, S.: Parabolic equations for curves on surfaces. II. Intersections, blow-up and generalized solutions. Ann. Math. (2) 133, 171-215 (1991)

3. Castro, I., Urbano, F.: Willmore Lagrangian surfaces of $\mathbb{C}^{2}$ and the Whitney sphere. Ann. Global Anal. Geom. 19, 153-175 (2001)

4. Ecker, K.: Regularity Theory for Mean Curvature Flow, Progress in Nonlinear Differential Equations and Their Applications, vol. 57. Birkhuser Boston, MA (2004)

5. Groh, K., Schwarz, M., Smoczyk, K., Zehmisch, K.: Mean curvature flow of monotone Lagrangian submanifolds. Math. Z. 257, 295-327 (2007)

6. Ilmanen, T.: Singularities of Mean Curvature Flow of Surfaces, preprint

7. Mantegazza,C.: Lecture notes on mean curvature flow. Progress in mathematics, vol. 290. Birkhäuser (2010)

8. Neves, A.: Singularities of Lagrangian mean curvature flow: zero-Maslov class case. Invent. Math. 168, 449-484 (2007)

9. Neves, A.: Singularities of Lagrangian mean curvature flow: monotone case. Math. Res. Lett. 17(1), 109-126 (2010)

10. Neves, A.: Finite time singularities for Lagrangian mean curvature flow. Ann. Math. 177, 1029-1076 (2013)

11. Oaks, J.: Singularities and self intersections of curves evolving on surfaces. Ind. Univ. Math. J. 43(3), 959-981 (1994)

12. Ros, A., Urbano, F.: Lagrangian submanifolds of $\mathbb{C}^{n}$ with conformal Maslov form and the Whitney sphere. J. Math. Soc. Jpn. 50, 203-226 (1998)

13. Schoen, R., Wolfson, J.: Minimizing area among Lagrangian surfaces: the mapping problem. J. Differ. Geom. 58, 1-86 (2001)

14. White, B.: A local regularity theorem for mean curvature flow. Ann. Math. 161, 1487-1519 (2005)

Publisher's Note Springer Nature remains neutral with regard to jurisdictional claims in published maps and institutional affiliations. 